\title{
Numerical study on dynamics of a nonspherical capsule in general flow
}

\author{
Huilin Ye ${ }^{\mathrm{a}}$, Haibo Huanga,*, Yi Sui ${ }^{\mathrm{b}}$, Xi-Yun Lu ${ }^{\mathrm{a}}$ \\ ${ }^{a}$ Department of Modern Mechanics, University of Science and Technology of China, Hefei, \\ Anhui 230026, China \\ ${ }^{b}$ School of Engineering and Materials Science, Queen Mary University of London, London \\ E1 4 NS, United Kingdom
}

\begin{abstract}
The dynamics of an oblate capsule in general flows is studied analytically and numerically. The capsule is modeled as a liquid-filled drop enclosed by a membrane. The governing equations for the dynamics of the capsule based on the Keller-Skalak (KS) theory and Skotheim-Secomb (SS) model are analytically derived. It is found that the capsule dynamics in general flows is controlled by two dimensionless parameters, the ratio of vorticity to strain rate of the flow and the ratio of the elastic force to fluid stress. In the literature, the transition between swinging (SW) and tumbling (TU) is always one way (TU to SW). As far as we know, it is the first time that the TU-SW-TU transition has been identified, i.e., the transition may also transfer from SW to TU after the transition (TU to SW) occurs under some circumstances. The possible mechanism is that the rotation of the flow suppresses the deformation along the vorticity direction of the capsule. The shape dynamics of a capsule and the rheology of dilute capsule suspension are also investigated.
\end{abstract}

Keywords: deformable particle, capsule dynamics, lattice Boltzmann, general flow, fluid structure interaction

\footnotetext{
* Corresponding author

Email address: huanghb@ustc.edu.cn (Haibo Huang)
} 


\section{Introduction}

Soft biological particles, such as vesicles, cells and capsules, have drawn much attention for many years and there are many theoretical, numerical, and experimental studies $[1,2,3,4,5,6,7,8]$. Detailed studies of a single particle are critical for understanding the rheology of bio-fluids. However, till today even behaviors of one single particle are not fully understood. A complete theoretical study in this topic is not easy. For example, the shape of the particle is not given a priori and continuously deforming. The shape evolution of the particle is determined by the dynamic balance between the interfacial forces and fluid stresses. To simplify the theoretical analysis, some studies $[9,10]$ constrained the number of degrees of freedom and others $[11,12]$ applied asymptotic analyses to investigate the dynamics. Due to the difficulties in theoretical study, there are many experimental $[5,13]$ or numerical studies $[2,14,15,16,17]$.

Capsule is a kind of unbreakable, hermetic membrane which is chemically or physically cross-linked. The membrane provides the resistance to shear and to change of volume. It has been shown that there is a steady tank-treading motion in simple shear flow when the capsule is initially spherical [14]. If the capsule is not initially spherical, a transition from tank-treading to tumbling may occur through reducing shear rate or increasing viscosity ratio [10, 15]. The swinging mode, which lies between tank-treading and tumbling, has been experimentally detected for red blood cells (RBCs) $[1,9]$. In this mode, the shape deformation is periodic and the inclination oscillates around a mean angle with respect to the direction of the flow. The swinging mode has also been found for capsule [15]. Due to the lacking of bending effect in capsule, the wrinkles would occur because of the compressive stress in the membrane that is imposed by the flow $[18,19,20]$.

Unlike capsules, vesicles exhibit a strong resistance to changes of volume, total surface area, and bending. Vesicles are not sensitive to strain. Hence, they can not show shear elasticity [15]. There are numerous theoretical and experimental studies on vesicles. In the KS theory [10], the particle is supposed 
to be a shape-fixed ellipsoid, which is enclosed by a inextensible membrane. And transition from tank-treading to tumbling mode is investigated. Fischer [21] introduced a shape memory model for the RBCs, in which, the rim of a red blood cell is always consisted of the same part of the membrane after relaxation from deformation. A regime of intermittent dynamics, characterized by several swinging cycles interrupt by a tumbling regularly near the transition from tanktreading to tumbling, was first observed by experiment [1]. Benefiting from the shape memory model [21], Abkarian et al. [1] and Skotheim et al. [9] introduced the energy barrier for the membrane based on the KS theory, and the model is able to predict the existence of the intermittent motion.

However, this intermittent motion is unable to be obtained in numerical simulations $[3,16,22,23]$. Considering the shape parameter, Noguchi [24, 25] predicted the synchronized rotation of phase angle and inclination angle with integer ratios of the frequencies. Vlahovska et al. [26] concluded that the intermittent behavior is a result of the shape-fixed assumption in the reduced models, in which only small-deformation is considered. They also pointed out that the intermittent behavior would be suppressed if there is a deformation along the vorticity direction. However, in the simple shear flow, the deformation along the vorticity direction is inevitable. Hence, the intermittent motion is difficult to be observed.

Although Abkarian et al. [1] observed the intermittent behavior, but the mechanism is not fully understood. Now days, numerical simulations become an important tool to study the intermittent dynamics. Cordasco et al. [27] and Peng et al. [28] showed that there is a large deformation along the vorticity direction during the TU-SW transition. More recently, Cordasco and Bagchi [4] present the computational evidence for the intermittent motion, which is characterized by an irregular sequence of TU interrupted by an SW, or vice versa, and synchronized motion with the cell rotation and membrane rotation with integer ratio of the rotational frequencies. The membrane in-plane elastic energy is introduced to explain these dynamics observed in the simulations [4].

However, the intermittent behavior research mentioned above are all based 
on the dynamics of vesicle and RBCs, in which the bending effects cannot be ignored [29]. In present work, we focus on capsule's intermittent dynamics without bending stiffness. Benefiting from the conclusion that the intermittent behavior would be suppressed by deformation along the vorticity direction [26], we introduce an important dimensionless parameter, the ratio of the vorticity to the strain rate [30]. The elastic energy form in the SS model [9] is based on the shape memory model, which is derived from experiment data. Here, an concrete energy form derived from the nonlinear membrane constitutive model is used in both our theoretical analysis and numerical simulations. It is expected to yield a better theoretical and numerical comparison. The capsule's membrane is assumed to follow the neo-Hookean (NH) law. It is found that the transition from TU to SW is no longer one way, i.e., an initial tumbling capsule may return to TU state after the TU-SW transition rather than eventually reach a stable swinging state.

The present work is intended to provide a better understanding of the behavior of an initially nonspherical capsule in general flow. In $\S 2$, the theoretical model and numerical method are introduced briefly. Next, in $\S 3$, our results, such as the mode transition, shape dynamics and rheology of dilute suspension are discussed and compared with the theoretical predictions. Finally, conclusions are presented in $\S 4$.

\section{Methods}

\subsection{Theoretical model}

In this section, our derivation follows the theoretical frame in $[9,10]$. The equations for the motion of capsules based on Jeffery's theory [31] have been derived by Keller and Skalak [10] analytically. They also improved the results of Roscoe [32], in which the inclination angle of the particle is assumed constant. Here, more general flows beyond simple shear flow are considered in our derivation, and the flow is still a plane flow. The undisturbed flow with a space-fixed 
(a)

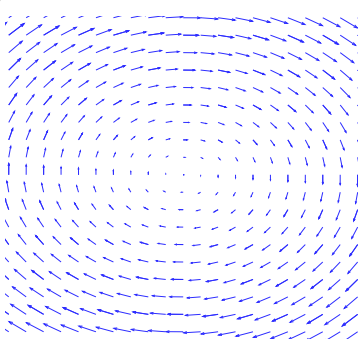

(b)

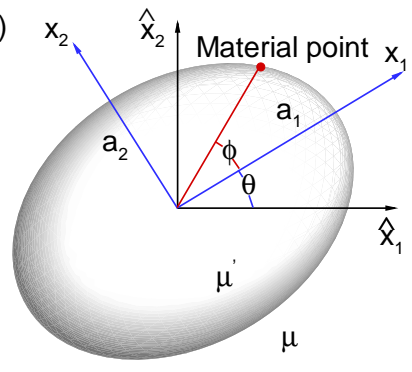

Figure 1: The schematic diagram of the model. (a) the velocity field of the flow with $\Xi=4$, (b) the membrane model of an ellipsoidal capsule in general flow.

frame shown in figure 1 is denoted by $\widehat{u}_{i}^{0}$, the velocity gradient is

$$
\delta_{i} u_{j}^{0}=s_{i j}-\epsilon_{i j k} \omega_{k}
$$

where $s_{i j}$ is the symmetric strain tensor, $\omega_{k}$ is the vorticity vector. $s=$ $\sqrt{\operatorname{tr}\left(s_{i j}^{2}\right) / 2}$ and $\omega=\left|\omega_{k}\right|$ are the strain rate and the vorticity, respectively. Here an important dimensionless parameter is defined as

$$
\Xi \equiv \omega / s
$$

which refers to the ratio of vorticity to strain rate. Then the velocity field of fluid is obtained as

$$
u_{1}^{0}=(s+\omega) \widehat{x}_{2}, u_{2}^{0}=(s-\omega) \widehat{x}_{1}, u_{3}^{0}=0 .
$$

Due to the moment balance of the capsule, the rate of change of the inclination angle $\theta$ is obtained as[10]

$$
\partial_{t} \theta=-\omega-\frac{2 a_{1} a_{2}}{a_{1}^{2}+a_{2}^{2}} \partial_{t} \phi+s \frac{a_{1}^{2}-a_{2}^{2}}{a_{1}^{2}+a_{2}^{2}} \cos 2 \theta .
$$

If the velocity and stress field shown in Appendix A are known, the internal dissipation $D$ and the rate of work $W_{p}$ done by the external fluid on the ellipsoid can be derived (refer to [10] for more details):

$$
D=V \mu^{\prime} f_{1}\left(\partial_{t} \phi\right)^{2}
$$




$$
W_{p}=V \mu\left(f_{2}\left(\partial_{t} \phi\right)^{2}+2 s f_{3} \partial_{t} \phi \cos 2 \theta\right)
$$

the parameters are defined in Appendix $\mathrm{A}$ and $V$ is the volume of the capsule. The work done by external fluid on the capsule is not only consumed by the internal dissipation, but also transferred to the elastic energy stored in the membrane [1, 9]. Suppose that the membrane follows the neo-Hookean law, which has the energy form [33]

$$
W^{N H}=\frac{1}{6} E\left(I_{1}-1+\frac{1}{I_{2}+1}\right),
$$

where $E$ is the Young's modulus, and $I_{1}=\lambda_{1}^{2}+\lambda_{2}^{2}-2, \quad I_{2}=\left(\lambda_{1} \lambda_{2}\right)^{2}-1$ are the first and second strain invariants, respectively. $\lambda_{1}$ and $\lambda_{2}$ are the principle strains. Here the elastic energy form is

$$
W^{N H}=\frac{2}{3} z_{1}^{2} E \sin ^{2} \phi,
$$

and the detailed derivation is shown in Appendix B.

The energy balance of the capsule is

$$
W_{p}=D+\partial_{t} \oint_{S_{c}} W^{N H},
$$

this yields

$$
V \mu\left(f_{2}\left(\partial_{t} \phi\right)^{2}+2 s f_{3} \partial_{t} \phi \cos 2 \theta=V \mu^{\prime} f_{1}\left(\partial_{t} \phi\right)^{2}+\frac{2}{3} E S_{c} z_{1}^{2} \sin 2 \phi \partial_{t} \phi,\right.
$$

where $S_{c}$ is the surface area of the capsule.

Combining equations (4) and (10) and scaling time with $t=T / 2 s$, it yields the dimensionless evolution equations for phase angle $\phi$ and inclination angle $\theta$,

$$
\begin{aligned}
& \partial_{T} \phi=h_{1}(\Lambda \sin 2 \phi+\cos 2 \theta), \\
& \partial_{T} \theta=-\frac{1}{2} \Xi-h_{2} \partial_{T} \phi+h_{3} \cos 2 \theta,
\end{aligned}
$$

where $h_{1}=-\frac{f_{3}}{f_{2}-\lambda f_{1}}, h_{2}=\frac{2 a_{1} a_{2}}{a_{1}^{2}+a_{2}^{2}}, h_{3}=\frac{a_{1}^{2}-a_{2}^{2}}{2\left(a_{1}^{2}+a_{2}^{2}\right)}$. If we defined $V=\frac{4}{3} \pi R_{0}^{3}$ and $S_{c}=(4 \pi+\Delta) R_{0}^{2}$, another important dimensionless parameter $\Lambda$ is defined as

$$
\Lambda \equiv-\frac{(4 \pi+\Delta) E z_{1}^{2}}{4 \pi R_{0} \mu f_{3} s} .
$$


Now the equations for the evolutions of phase angle $\phi$ and inclination angle $\theta$ are derived. They are totaly characterized by three dimensionless parameters $\lambda, \Xi$ and $\Lambda$ under the assumption that the capsule preserves an undeformed shape. In present work, the main task is to study the influence of vorticity on the dynamics of the capsule. Because the viscosity ratio effects have been studied extensively $[10,22,25,34]$, the viscosity ratio $\lambda$ is set to be unity in our study. We focus on the effects of $\Xi$ and $\Lambda$.

\subsection{Numerical method}

(a)

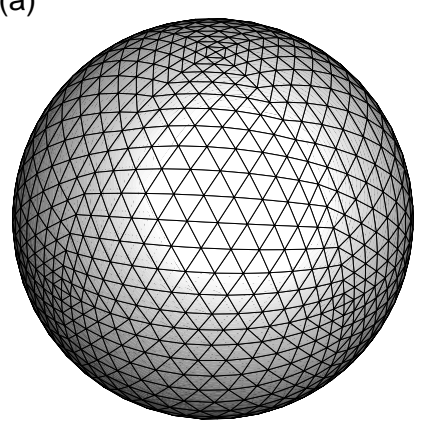

(b)

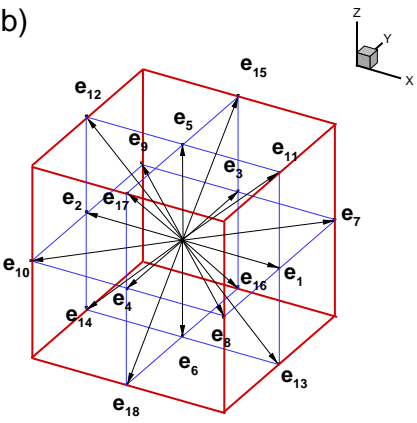

Figure 2: (a) Discretization of a sphere; (b) D3Q19 model.

\subsubsection{Finite element membrane model}

In our study, the membrane dynamics is numerically solved by finite element method, in which the neo-Hookean constitutive law (7) is adopted. The 3D capsule membrane is discretized into flat triangular elements. To discretize the unstressed interface, each triangular face of a regular octahedron is subdivided into $4^{n}$ triangular elements. These elements are then projected radically onto a sphere. The geometry of each element is described by its three vertices. The discretization of a sphere surface is shown in figure 2(a). For oblate spheroid, it is necessary to make the coordinates multiply by the aspect ratio of the oblate spheroid. 
First, the problem is reduced to a $2 \mathrm{D}$ (planar) deformation by transforming the undeformed and deformed surface elements to a common plane using rigidbody rotations, the detailed method is given in [33]. Here gives the in-plane displacements $\mathbf{v}$ of the vertices and the displacement gradient tensor $\mathbf{D}$. Then the in-plane principal strains are:

$$
\lambda_{i}^{2}=\frac{1}{2}\left[G_{11}+G_{22} \pm \sqrt{\left(G_{11}-G_{22}\right)^{2}+4 G_{12}^{2}}\right], i=1,2,
$$

where $\mathbf{G}=\mathbf{D}^{T} \mathbf{D}$. The elastic forces acting on the three vertices of a triangular element are obtained from the strain energy function $W^{N H}$ using the principal of virtual work as:

$$
\mathbf{f}\left(\mathbf{x}^{\prime}, t\right)=-\frac{\partial W^{N H}}{\partial \mathbf{x}^{\prime}} .
$$

Then, the in-plane force $\mathbf{f}^{p}$ can be obtained as

$$
\mathbf{f}^{p}=\frac{\partial W^{N H}}{\partial \lambda_{1}} \frac{\partial \lambda_{1}}{\partial \mathbf{v}}+\frac{\partial W^{N H}}{\partial \lambda_{2}} \frac{\partial \lambda_{2}}{\partial \mathbf{v}} .
$$

Because each node of the discrete membrane belongs to more than one element, the resultant force on a node is the sum of the forces exerted by the m elements attached to the node. So far, the force calculated is the fluid force acting on the capsule membrane. Its equal and opposite counterpart is the force acting on the fluid. It is distributed to the surrounding fluid by the approach described by the immersed boundary method which will discussed below.

\subsubsection{Multi-block lattice Boltzmann method}

The general flow is solved by Lattice Boltzmann method (LBM), which is an efficient solver for the Navier-Stokes (NS) equations with a low Reynolds number. Here, the D3Q19(see fig 2(b)) model is used, in which the discrete lattice Boltzmann equation (LBE) has the form of:

$$
f_{i}\left(\mathbf{x}+\mathbf{e}_{i} \Delta t, t+\Delta t\right)=f_{i}(\mathbf{x}, t)-\frac{1}{\tau}\left(f_{i}(\mathbf{x}, t)-f_{i}^{e q}(\mathbf{x}, t)\right),
$$

where $f_{i}(\mathbf{x}, t)$ is the distribution function for particles with velocity $\mathbf{e}_{i}$ at position $\mathbf{x}$ and time $t, \Delta t$ is the lattice time interval, $f_{i}^{e q}(\mathbf{x}, t)$ is the equilibrium distribution function and $\tau$ is the non-dimensional relaxation time. 
In the D3Q19 model, the fluid particles have the possible discrete velocities stated as follows[33, 35]:

$$
\begin{aligned}
& {\left[\mathbf{e}_{0}, \mathbf{e}_{1}, \mathbf{e}_{2}, \mathbf{e}_{3}, \mathbf{e}_{4}, \mathbf{e}_{5}, \mathbf{e}_{6}, \mathbf{e}_{7}, \mathbf{e}_{8}, \mathbf{e}_{9}, \mathbf{e}_{10}, \mathbf{e}_{11}, \mathbf{e}_{12}, \mathbf{e}_{13}, \mathbf{e}_{14}, \mathbf{e}_{15}, \mathbf{e}_{16}, \mathbf{e}_{17}, \mathbf{e}_{18}\right]=} \\
& {\left[\begin{array}{ccccccccccccccccccc}
0 & 1 & -1 & 0 & 0 & 0 & 0 & 1 & 1 & -1 & -1 & 1 & -1 & 1 & -1 & 0 & 0 & 0 & 0 \\
0 & 0 & 0 & 1 & -1 & 0 & 0 & 1 & -1 & 1 & -1 & 0 & 0 & 0 & 0 & 1 & 1 & -1 & -1 \\
0 & 0 & 0 & 0 & 0 & 1 & -1 & 0 & 0 & 0 & 0 & 1 & 1 & -1 & -1 & 1 & -1 & 1 & -1
\end{array}\right]}
\end{aligned}
$$

The equilibrium distribution function $f_{i}^{e q}(\mathbf{x}, t)$ can be calculated as:

$$
f_{i}^{e q}(\mathbf{x}, t)=\omega_{i} \rho\left[1+\frac{\mathbf{e}_{i} \cdot \mathbf{u}}{c_{s}^{2}}+\frac{\left(\mathbf{e}_{i} \cdot \mathbf{u}\right)^{2}}{2 c_{s}^{4}}-\frac{(\mathbf{u})^{2}}{2 c_{s}^{2}}\right],
$$

where the weighting coefficients $\omega_{i}=1 / 3(i=0), \omega_{i}=1 / 18(i=1-6), \omega_{i}=$ $1 / 36(i=7-18)$. The term $c_{s}$ represents the sound speed which equals $\Delta x /(\sqrt{3} \Delta t)$.

The relaxation time is related to the kinematic viscosity $\nu$ in Navier-Stokes equation in the form of

$$
\nu=\left(\tau-\frac{1}{2}\right) c_{s}^{2} \Delta t
$$

Once the particle density distribution is known, the fluid density and momentum are calculated as

$$
\rho=\sum_{i} f_{i}, \quad \rho \mathbf{u}=\sum_{i} f_{i} \mathbf{e}_{i} .
$$

Here the multi-block lattice Boltzmann method proposed by Yu and Girimaji is employed [36]. We consider a two-block system, the computational domain is divided into two blocks which are connected through the interface. The lattice space ratio between coarse and fine grids equals two. The capsule is immersed in the fine mesh block. On the interface between the two blocks, the exchange of variables follows a certain relation so that the mass and momentum are conserved and the stress is continuous across the interface. Detailed method is explained in [33]. 


\subsubsection{Immersed boundary method}

In our study, the immersed boundary method is adopted to couple the finite element model with lattice Boltzmann method, in which a force density is distributed to the Cartesian mesh in the vicinity of the moving boundary in order to account for the effect of the boundary. The fluid domain is represented by Eulerian coordinates $\mathbf{x}$, while the boundary of the capsule is represented by Lagrangian coordinates $\mathbf{s}$. Any position on the capsule membrane can be written as $\mathbf{X}(\mathbf{s}, t)$. The term $\mathbf{F}(\mathbf{s}, t)$ represents the membrane force density induced by capsule deformation, and the term $\mathbf{f}(\mathbf{x}, t)$ represents the fluid body force density.

In order to satisfy the no-slip boundary condition, the flexible membrane should move at the same velocity as the fluid around it. That is

$$
\frac{\partial \mathbf{X}(\mathbf{s}, t)}{\partial t}=\mathbf{u}(\mathbf{X}(\mathbf{s}, t))
$$

This condition will cause the capsule to deform. The membrane force density $\mathbf{F}(\mathbf{s}, t)$ is obtained by the finite element membrane model discussed in 2.2.1, and is distributed to the fluid mesh points near it by

$$
\mathbf{f}(\mathbf{x}, t)=\int \mathbf{F}(\mathbf{s}, t) \delta(\mathbf{x}-\mathbf{X}(\mathbf{s}, t)) d \mathbf{s}
$$

where $\delta$ is a smoothed approximation of the Dirac Delta function. In the present $3 \mathrm{D}$ study, it is chosen to be:

$$
\delta(\mathbf{x}-\mathbf{X}(\mathbf{s}, t))=\delta(x-X(\mathbf{s}, t)) \delta(y-Y(\mathbf{s}, t)) \delta(z-Z(\mathbf{s}, t)),
$$

where

$$
\delta(r)= \begin{cases}\frac{1}{4}\left(1+\cos \left(\frac{\pi|r|}{2}\right)\right), & r \leq 2 \\ 0, & r>2\end{cases}
$$

and the position of a Lagrangian node in the Eulerian coordinates is $\mathbf{X}=$ $(X, Y, Z)$.

The same approximation function is used to obtain the velocities of the Lagrangian nodes on the moving boundary. The mathematical form can be written as follows:

$$
\frac{\partial \mathbf{X}}{\partial t}=\int \mathbf{u}(\mathbf{x}, t) \delta(\mathbf{x}-\mathbf{X}(\mathbf{s}, t)) d \mathbf{x}
$$


To take into account the fluid body force density $\mathbf{f}(\mathbf{x}, t)$, an extra term $F_{i} \Delta t$ should be added into the right hand side of the LBE (Eq.(16)) [37], i.e.,

$$
F_{i} \Delta t=\left(1-\frac{1}{2 \tau}\right) \Delta t \omega_{i}\left[\frac{\mathbf{e}_{i}-\mathbf{u}}{c_{s}^{2}}+\frac{\left(\mathbf{e}_{i} \cdot \mathbf{u}\right)}{c_{s}^{4}} \mathbf{e}_{i}\right] \cdot \mathbf{f} .
$$

Correspondingly, the equation for momentum of the fluid i.e., Eq.(20) should be replaced by

$$
\rho \mathbf{u}=\sum_{i} f_{i} \mathbf{e}_{i}+\frac{1}{2} \mathbf{f} \Delta t
$$

The validations of this method, such as the grid-independence and time stepindependence studies, and deformation of capsule in simple shear flow, have been presented in details in $[16,33]$. Here, the computational domain is a cubic box with side $10 R_{0}$ which is large enough to neglect the boundary effect. The grid resolutions in the fine and coarse blocks are $\Delta x_{f}=\Delta y_{f}=\Delta z_{f}=R_{0} / 12$ and $\Delta x_{c}=\Delta y_{c}=\Delta z_{c}=R_{0} / 6$, respectively. The capsule is discretized into 8192 triangular elements connecting 4098 nodes. The capsule is initially oblate with

shape parameter $r_{2}=\frac{10}{11}$ unless it is specially stated, the initial inclination angle is $\frac{\pi}{4}$. The Taylor deformation parameter $D_{x y}$ is

$$
D_{x y}=\frac{L-B}{L+B},
$$

where $L$ and $B$ are semi-major and semi-minor axes, respectively. The time is nondimensionalized by $\frac{1}{2 s}$.

\section{Results and discussion}

\subsection{Shape dynamics of a capsule in general flow}

It is noted that in our derivation, i.e., Eq. (11), the shape of the capsule is supposed to be fixed. However, the shape will change continuously in the numerical simulation. Hence, the numerical results will be slightly different from the theoretical results. In the follows, effects of the two dimensionless parameters $\Xi$ and $\Lambda$ on the deformation of the capsule are discussed in detail.

From figure 3 (a) and (b), it is seen that for a fixed $\Lambda$, the deformation of capsule decreases with the increase of $\Xi$. The effect of $\Xi$ is similar to the effect 

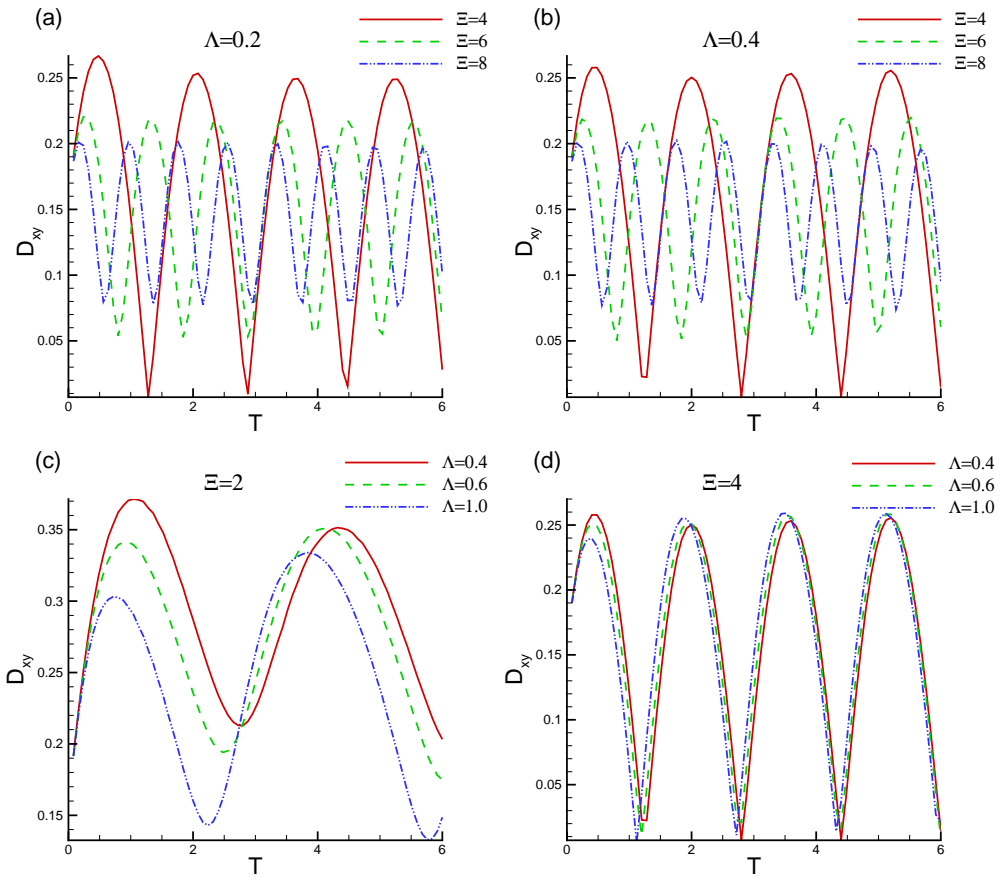

Figure 3: The evolution of the capsule: (a) $\Lambda=0.2$ and (b) $\Lambda=0.4$ with different $\Xi$, (c) $\Xi=2$ and (d) $\Xi=4$ with different $\Lambda$

of viscosity ratio $\lambda$ [10]. It is also found that a larger $\Xi$ results in a shorter period of the deformation. Because $\Lambda$ is the ratio of elastic stress to viscous force of the fluid exerting on the capsule, it dominates the deformation of the capsule.

The effect of $\Xi$ is similar to that of $\lambda$ but with some differences. In the effect of $\lambda$, increasing $\lambda$ makes the capsule more viscous and the dynamic force in the membrane increases even the strain rate $s$ is fixed. However, the increasing of $\Xi$ results in the acceleration of the rotation of the flow and it can not change the dynamic force if the strain rate $s$ is fixed [23]. Because the deformation of the capsule would need time to evolve, when $\Xi$ is larger, the evolution period is reduced and the deformation magnitude is suppressed. That is shown in figure 3 (a) and (b). 
The period of the deformation is mainly determined by $\Xi$. Through many tests with different $\Lambda$ and $\Xi$, it is found that the period of deformation $T_{D}$ times $\Xi$ is a constant, i.e.,

$$
T_{D} \Xi=1 / C_{0}
$$

where $C_{0}=0.15625$. Hence, $2 \pi /\left(\Xi T_{D}\right)=2 \pi C_{0}=1$. This is also supported by cases of $\Xi=4$ and $\Xi=8$ in figure 3 (a) and (b). It is seen that the period in the case of $\Xi=4$ is two times of that in the case $\Xi=8$.

From figure 3 (c), it is seen that when $\Xi$ is relatively small, the deformation magnitude decreases with the increase of $\Lambda$. However, from figure 3 (d), we can see that when $\Xi$ is large, the deformation is almost independent of $\Lambda$. As the explanation mentioned above, if $\Xi$ is large enough, capsule would experience the same deforming procedure no matter what $\Lambda$ is. In other words, as $T_{D}$ is inversely proportional to $\Xi$, if $\Xi$ is large enough to suppress the deformation procedure of the case with the largest $\Lambda$, then all of the cases with different $\Lambda$ would undergo the same suppressed procedure.

We give a brief theoretical explanation to the influence of $\Xi$ on the deformation of the capsule. Here, following [26], we only consider a nearly spherical capsule, the radial position $r_{s}$ of the surface of the capsule membrane can be expressed by

$$
r_{s}=1+\varepsilon f(\vartheta, \varphi, t)
$$

where $f$ is the deviation of the capsule shape from a spherical shape. $f$ is expanded into series of scalar spherical harmonics $Y_{j m}$,

$$
f=\sum_{j=2}^{\infty} \sum_{m=-j}^{j} f_{j m} Y_{j m} .
$$

We add the rotational part of the flow to the governing equation (3.16) in [26], then it can be written as

$$
\frac{\partial f_{22}}{\partial T}=i \Xi f_{22}-i \Gamma-\Pi\left(f_{22}-g_{22}\right),
$$

where $\Gamma=\frac{8 \sqrt{30 \pi}}{(23 \lambda+32) \sqrt{\Delta}}, \Pi=\frac{16 E}{6(23 \lambda+32) \mu s R_{0}}$ and $g_{22}$ is the initial shape function 


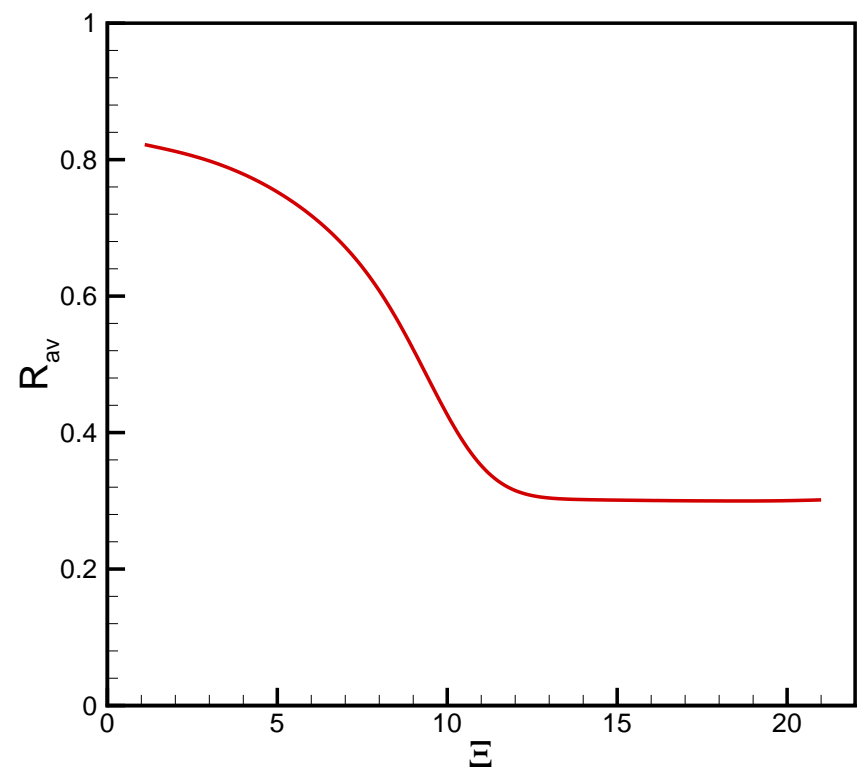

Figure 4: The average value of $R$ over one period as a function of $\Xi$ when $\Lambda=0.2$

with the same definition of $f_{22}$. If we set

$$
f_{22}=\operatorname{Rexp}(-2 i \theta)
$$

we can obtain the shape evolution equation

$$
\frac{\partial R}{\partial T}=\Gamma \sin 2 \theta-\Pi R+\frac{1}{2} \Pi \sqrt{\Delta} \cos (2 \phi-2 \theta) .
$$

We use the result of equation (11) to obtain the value of $\theta$ and $\phi$, then the shape equation is solved by numerical method. The result of $R$ as a function of $\Xi$ is shown in figure 4 with $\Lambda=0.2$.

The Taylor deformation parameter can also been written as a function of $R$ [38]

$$
D_{x y} \approx \frac{R}{8} \sqrt{\frac{15 \Delta}{\pi}} .
$$

From figure4, we can see $R$ decreases with the increase of $\Xi$ when $\Xi$ is not large, which means that increasing $\Xi$ suppresses the deformation of the capsule. 


\subsection{Intermittent dynamics}

The normalized mean tumbling rate is defined as $[23,38]$

$$
\langle\dot{\theta}\rangle \equiv \frac{\left\langle\partial_{T} \theta\right\rangle}{\left\langle\partial_{T} \theta\right\rangle+\left\langle\partial_{T} \phi\right\rangle},
$$

where

$$
\begin{aligned}
& \left\langle\partial_{T} \theta\right\rangle \equiv \lim _{t \rightarrow \infty} \frac{1}{t} \int_{0}^{t} \partial_{T} \theta(T) d T, \\
& \left\langle\partial_{T} \phi\right\rangle \equiv \lim _{t \rightarrow \infty} \frac{1}{t} \int_{0}^{t} \partial_{T} \phi(T) d T .
\end{aligned}
$$

In a pure tumbling motion, the inclination angle $\theta$ grows continuously while the phase angle $\phi$ oscillates, which implies the mean tumbling rate $\langle\dot{\theta}\rangle=1$ for a large time $t$. On the contrary, in a pure swinging motion, the phase angle $\phi$ grows without bounds while the inclination angle $\theta$ oscillates, which implies

$\langle\dot{\theta}\rangle=0$ for a large $t$. Obviously, the intermittent regime corresponds to a mean tumbling rate between 0 and 1 .

Here, different dynamic modes are identified. The phase diagram of different regimes spanning on the $(\Xi, \Lambda)$ plane is shown in figure 5 . Three grey scale regimes represent $\mathrm{SW}$, Intermittent, TU modes, which are obtained through solving Eq.(11) by fourth order Runge-Kutta scheme. The lines represent boundaries between different regimes.

The numerical simulation results are also shown in figure 5 . The symbols and the dashed lines are the modes and boundaries of different modes, respectively. Blue filled circles, green filled squares, and red filled triangles represent SW, TU, and TU-SW transition modes, respectively. Pink filled diamonds denote the new mode that we find: TU-SW-TU mode. It is seen that the boundaries predicted theoretically are not consistent with those obtained from the numerical results. However, it is not surprise because in the present theoretical predictions the capsule shape is initially fixed. The theoretical predictions will be close to the numerical results if the initial shape is replaced by the deformed shape in the theoretical model $[23,38]$. Because the shape deformation as a function of time depends on not only $\Lambda$ but also $\Xi$, it is still difficult to theoretically analyse the deformation $[24,25]$. 


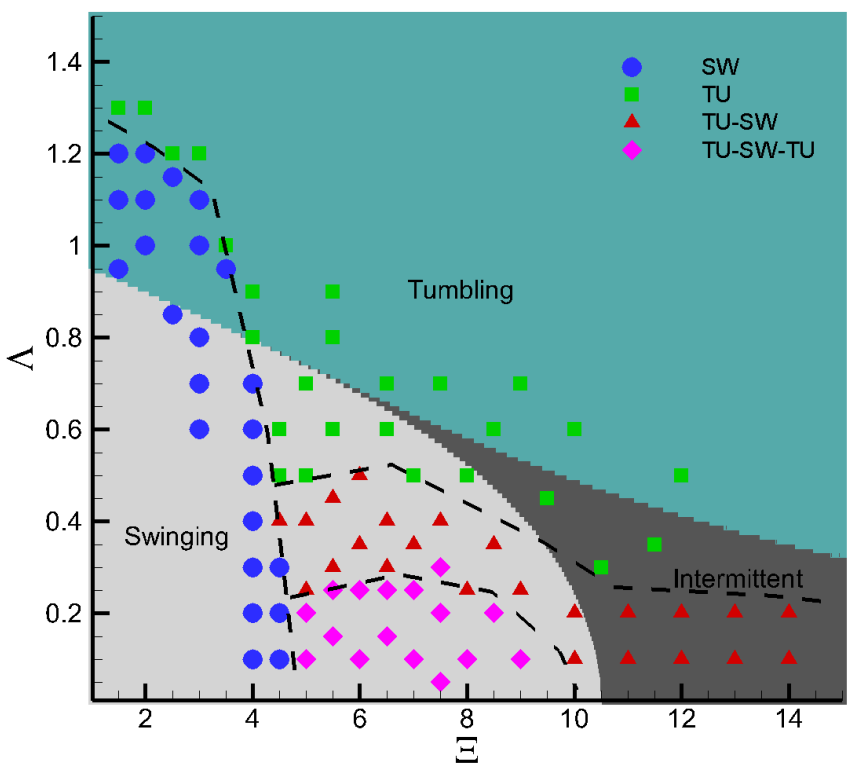

Figure 5: Phase diagram of the capsule dynamical states in general flow. The grey scales represent the three regimes obtained by theoretical model, the solid lines indicate their boundaries. The symbols and the dashed lines represent numerical results. They are the modes and boundaries of different modes, respectively. Blue filled circles, green filled squares, and red filled triangles represent SW, TU, and TU-SW transition modes, respectively. Pink filled diamonds denote TU-SW-TU mode.

From the figure 5 we see the profile of the distribution of the modes is similar to the results of [38], in which the horizontal axis is the viscosity ratio. Here we present some connections between the viscosity ratio $\lambda$ and dimensionless parameter $\Xi$. If Equation (11) is divided by $-h_{1}$, it yields

$$
\begin{aligned}
& \partial_{\tau} \phi=-(\Lambda \sin 2 \phi+\cos 2 \theta), \\
& \partial_{\tau} \theta=-\frac{1}{2} \Xi \lambda^{*}-h_{2} \partial_{\tau} \phi+h_{3} \lambda^{*} \cos 2 \theta,
\end{aligned}
$$

where $h_{1}=-\frac{1}{\lambda^{*}}, \tau=T / \lambda^{*}$. if we set $\Xi=1$, it would reduce to the expression in [38]. From Equation (38), it is seen that there are two terms in the evolution equation of $\theta$ containing $\lambda^{*}$. However, there is only one term including the 
parameter $\Xi$. To analyze contributions of these two terms, first we assumed that $\Xi \lambda^{*}$ is a constant. If $\lambda^{*}$ is small and $\Xi$ is large, then contribution of the third term in the RHS of the second equation in Eq.(38) is minor compared to the first term. Under this circumstance, the two cases that only changing $\lambda^{*}$ and only changing $\Xi$ contribute equivalently to the equation. Hence, in the region of large $\Xi$, the phase (mode) distributions in the phase diagram that we obtained (see figure 5 ) is consistent with those in the literature [3, 22, 23].

If $\lambda^{*}$ is large $\left(\Xi \lambda^{*}=\right.$ const $)$, then contribution of the last term in the RHS of Eq.(38) is comparable to the first term. The two cases that only changing $\lambda^{*}$ and only changing $\Xi$ are different, then it would lead to different results for the evolution of $\theta$. In this situation, $\Xi$ is not large enough, the phase distribution in the phase diagram is different from those in previous studies, which is discussed following.

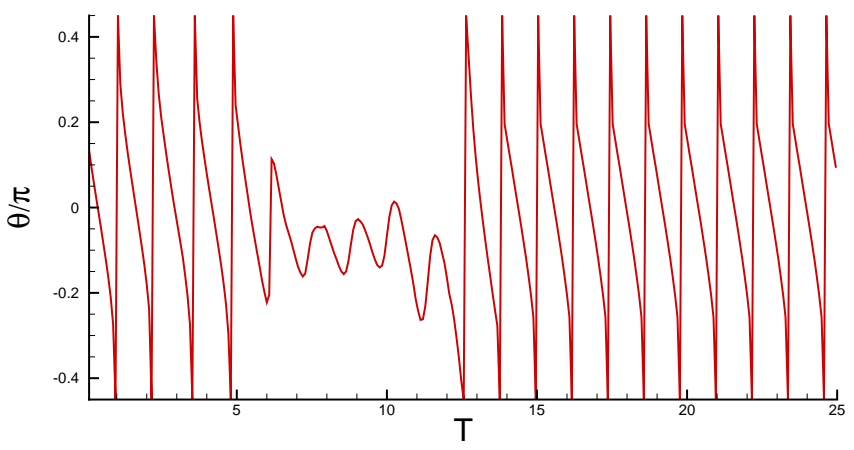

Figure 6: The evolution of inclination angle with $\Xi=5, \Lambda=0.1$

Here we focus on the new transition mode of TU-SW-TU, which is shown in figure 6 with the parameters $\Xi=5, \Lambda=0.1$. In the transition mode, an initially tumbling mode transfers to swinging mode, and then it would return to a tumbling mode, and within our simulation time $(T=40)$ it will maintain this steady tumbling mode. In [3] and [22], the transition is always one way, in which the capsule would maintain a stable swinging mode after transition from tumbling, and this cannot reverse. Recently, Cordasco et al. [4] proposed 
a mechanism that the membrane in-plane elastic energy is relevant to the intermittent motion of RBC. However, here the capsule is an ellipsoid particle which is different from the RBC with circular biconcave discoid shape, such mechanism does not work.

Now we provide an explanation for the observed dynamics in numerical simulations. As shown in figure 5, the region of the TU-SW-TU transition is constricted to a range with a moderate $\Xi$ and low $\Lambda$. The energy barrier theory reveals that the capsule with lower $\Lambda$ would be more likely to overcome the energy barrier due to large hydrodynamic shear force. However, the conclusion is based on the assumption that the deformation is large when the capsule has a lower $\Lambda$. Here, the deformation is small at a lower $\Lambda$. As discussed in Sec. 3.1, large $\Xi$ would confine the deformation, which implies that the deformation along vorticity direction is constant or changing little due to volume preservation. That is consistent with the explanation of [26]. In other words, at this range, the effect of constraint on the deformation along vorticity direction is dominated, which is totally different from the energy barrier mechanism.

When $\Xi$ is moderate (approximately $\Xi \approx 6$ ), the capsule with smaller $\Lambda$ deforms less than that in the cases of $\Xi=1$, which is a purely shear flow. In the cases of smaller $\Lambda$, the suppression to the deformation is stronger. So, the capsule with small $\Lambda$ is more likely to transit to tumbling mode comparing to the capsules with large $\Lambda$. While when $\Xi$ becomes larger, the deformations are almost identical and independent of $\Lambda$. Under this circumstance, the deformation would not affect the transition. On the condition, the energy barrier theory is valid, in which when $\Lambda$ is low, the fluid shear stress acting on the capsule is sufficient to force the membrane to tank tread [9].

\subsection{Effect of initial aspect ratio}

In simple shear flow, the effect of initial aspect ratio has been studied in $[10,15]$ and it has been found that a capsule deviating largely from spherical profile is more likely to transit to tumbling mode. Figure 7 shows the aspect ratio effect on the transition dynamics in our study. From figure 7 , it is seen that 
(a)

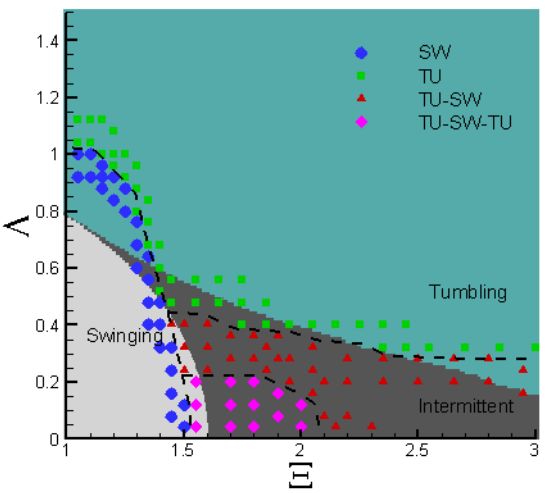

(b)

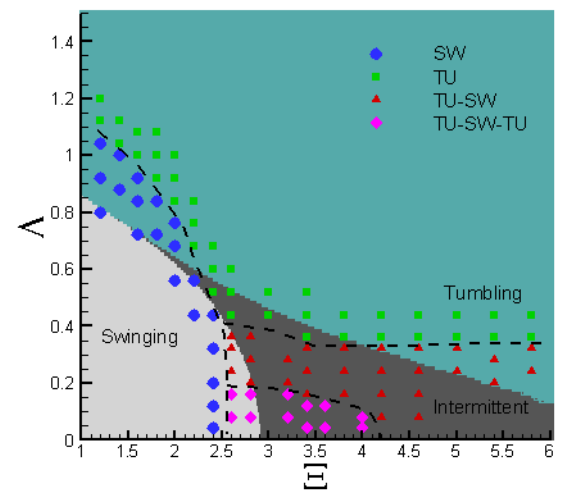

Figure 7: Phase diagrams for capsule dynamic states with different initial aspect ratio. (a) $r_{2}=0.5$ and (b) $r_{2}=0.7$

in the simple shear flow $(\Xi=1)$, capsules with small initial aspect ratio could transit to tumbling motion with softer membrane (lower $\Lambda$ ), which is consistent with that observed in $[15,10]$. Actually not only $\Xi=1$ but also the other $\Xi>1$, the similar situation can be observed, i.e., for cases with smaller initial aspect ratio, transition to tumbling motion becomes easier (with lower $\Lambda$ ) (see figure 7).

From figure 7 , it is also seen that for the same $\Lambda$, a capsule with smaller initial aspect ratio would transit to tumbling motion with a lower $\Xi$. Here $\Xi$ represents the rotational strength of the fluid. Hence, the capsule needs less rotation effect of the fluid to transit to tumbling mode. For the TU-SW and TU-SW-TU transition modes, the situation is similar, i.e., less rotation effect is required for cases with smaller initial aspect ratio. Hence, the boundaries between different modes are all shifted to lower $\Xi$ and $\Lambda$ regime for the capsule with smaller initial aspect ratio.

\subsection{Rheology of dilute suspension}

Instead of the dimensionless particle stress tensor [2, 39], here the intrinsic viscosity $[34,40]$ is used to evaluate the contribution of the capsules on the bulk 
rheology.

$$
[\eta]=\frac{\eta_{e f f}-1}{\Omega},
$$

where $\eta_{e f f}$ is the dimensionless effective viscosity defined as $\eta_{e f f}=\frac{\bar{\sigma}_{12}}{2 e_{12}^{0}}$, and $\Omega$ is the concentration. Substituting Eq.(A.2) into Eq.(39), the intrinsic viscosity can be calculated as

$$
\begin{aligned}
{[\eta] } & =\frac{\mu A_{12}^{*}}{2 e_{12}^{0} \Omega}=\frac{4 \mu}{\Omega} \frac{g_{1} e_{12}^{*}-\alpha_{2}^{2} g_{3}^{\prime}\left(\zeta_{12}^{*}-\varepsilon_{12 k} \dot{\theta}\right)}{g_{3}^{\prime}\left(\alpha_{1}^{2} g_{1}+\alpha_{2}^{2} g_{2}\right)} \frac{1}{2 s \cos (2 \theta)} \\
& =\frac{4 \mu}{\Omega} \frac{1}{J}\{M-N(\Lambda \sin 2 \phi+\cos 2 \theta)\},
\end{aligned}
$$

where $J=g_{3}^{\prime}\left(\alpha_{1}^{2} g_{1}+\alpha_{2}^{2} g_{2}\right), M=\frac{1}{2} g_{1}+\alpha_{2}^{2} g_{3}^{\prime} h_{3}$ and if we set $\tan \alpha=\frac{a_{1}^{2}-a_{2}^{2}}{2 a_{1} a_{2}}$, then $N=h_{1}\left(g_{1} \tan \alpha+\frac{\alpha_{2}^{2} g_{3}^{\prime}}{\cos \alpha}-h_{2} \alpha_{2}^{2} g_{3}^{\prime}\right)$. It seems that equation (8) doesn't contain the parameter $\Xi$, which indicates that the rotation of the fluid flow has no contribution to the bulk rheology of the flow. It can be understood as follows. The hydrodynamic stress of a purely rotating fluid vanishes, and it cannot induce forces at the membrane. Then according to the definition of the bulk rheology [39, 2], the rotation has no contribution to the bulk rheology.

However, $\Xi$ still plays an important role in the rheology of the suspension through the shape dynamics effect because $J, M$ and $N$ are all functions of shape parameters. Hence, they should be functions of $\Xi$. This result of a dilute suspension $(\Omega \ll 1)$ is shown in figure 8 . The mean value of intrinsic viscosity, $[\bar{\eta}]$, is computed by averaging $[\eta]$ over one period. It is seen that for a specific $\Xi$, $[\bar{\eta}]$ increases with $\Lambda$. Hence, the suspension with deformable capsules exhibits shear thinning behavior, which is consistent with [34].

It is also found that $[\bar{\eta}]$ decreases with $\Xi$ for the same $\Lambda$. Here, for a given $\Lambda$, a larger $\Xi$ could shorten the period of the disturbance of the capsule on the flow, i.e., the $\Xi$ suppresses the disturbance. Gao et al. [34] proposed that if the particles in the suspension disturb the flow less, the intrinsic viscosity may decrease. Hence, $[\bar{\eta}]$ would become smaller. It is also seen from figure 8 that $[\bar{\eta}]$ may be less than 0 , which indicates that the capsule is softer than the fluid in 


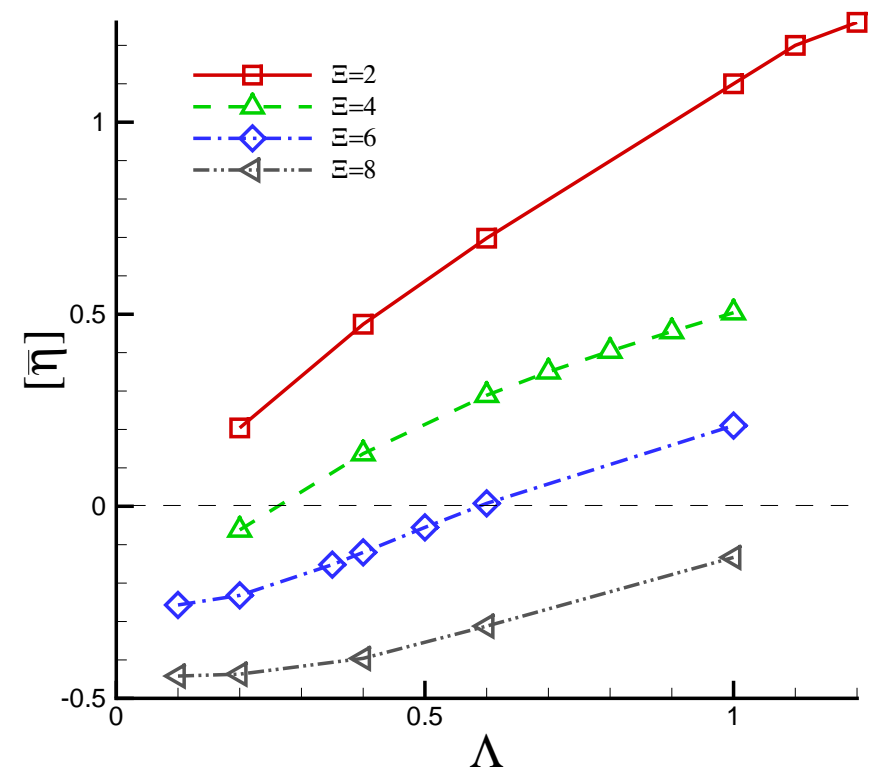

Figure 8: The mean intrinsic viscosity $[\bar{\eta}]$ as a function of $\Lambda$ for cases $\Xi=2,4,6,8$.

some special conditions [34].

\section{Conclusion}

We have investigated the dynamics of a nonspherical capsule in general flow using theoretical and numerical analyses. The numerical results have discrepancies with the theoretical results due to the fixed shape assumption in the theoretical analysis. On the other hand, we focus on analyzing the transition dynamics. Two transition behaviors (the TU-SW transition and TU-SW-TU transition ) are found in the intermittent regime, which is different from the intermittent behavior predicted by theoretical models. To the best of our knowledge, the TU-SW-TU transition has not been observed in capsule dynamics up to now and it is the first time that we obtained this transition in general flow. The mechanism for the TU-SW-TU transition may be associated with the deformation along the vorticity direction. The effects of $\Xi$ (the ratio of vorticity 
to the strain rate) on the deformation of capsule is demonstrated. It seems that $\Xi$ suppresses the deformation in the vorticity direction, which triggers the TU-SW-TU transition. Also, the effect of initially aspect ratio of the capsule is investigated. The result shows that a capsule deviating largely from spherical profile is more likely to transit to tumbling mode, which is similar to the situation of a capsule in simple shear flow. Finally, the intrinsic viscosity as functions of the two dimensionless parameters $\Lambda$ and $\Xi$ is studied by theoretical and numerical analyses. A deformable capsule would display a shear thinning behavior, and the vorticity of the flow would strengthen this shear thinning effect.

\section{ACKNOWLEDGMENTS}

Huang is supported by National Natural Science Foundation of China (Grant No. 11172297), Anhui Provincial Natural Science Foundation (Grant No. 130808 5MA06), and Program for New Century Excellent Talents in University, Ministry of Education, China (NCET-12-0506).

\section{Appendix A. Stress field of the system}

The velocity field of the membrane in the body-fixed frame is assumed to be

$$
u_{1}^{m}=\nu\left(-a_{1} / a_{2}\right) x_{2}, u_{2}^{m}=\nu\left(a_{2} / a_{1}\right) x_{1}, u_{3}^{m}=0,
$$

where $\nu$ is defined as $\nu=\partial \phi / \partial t$. $\phi$ is phase angle shown in figure 1 . Then the stress tensor can be written as

$$
\sigma_{i j}=-p \delta_{i j}+\mu\left(A_{i j}^{*}+2 e_{i j}^{m}\right)
$$

where $p$ is an arbitrary constant pressure and $e_{i j}^{m}$ is defined as

$$
e_{i j}^{m}=\frac{1}{2}\left(u_{j, i}^{m}+u_{i, j}^{m}\right) \text {. }
$$

The tensor $A_{i j}^{*}$ are independent of $x_{i}$, two elements of $A_{i j}^{*}$ are

$$
\left\{\begin{array}{l}
A_{11}^{*}=\frac{4}{3} \frac{2 g_{1} e_{11}^{*}-g_{2} e_{22}^{*}-g_{3} e_{33}^{*}}{g_{2}^{\prime \prime} g_{3}^{\prime \prime}+g_{3}^{\prime \prime} g_{1}^{\prime \prime}+g_{1}^{\prime \prime} g_{2}^{\prime \prime}} \\
A_{12}^{*}=4 \frac{g_{1} e_{12}^{*}-\alpha_{2}^{2} g_{3}^{\prime}\left(\zeta_{12}^{*}-\epsilon_{123} \theta\right)}{g_{3}^{\prime}\left(\alpha_{1}^{2} g_{1}+\alpha_{2}^{2} g_{2}\right)}
\end{array}\right.
$$


where

$$
\begin{gathered}
e_{i j}^{*}=e_{i j}^{0}-e_{i j}^{m}, \\
e_{i j}^{0}=\frac{1}{2}\left(u_{j, i}^{0}+u_{i, j}^{0}\right),
\end{gathered}
$$

and

$$
\left\{\begin{array}{l}
\zeta_{i j}^{*}=\zeta_{i j}^{0}-\zeta_{i j}^{m}, \\
\zeta_{i j}^{0}=\frac{1}{2}\left(u_{j, i}^{0}-u_{i, j}^{0}\right), \\
\zeta_{i j}^{m}=\frac{1}{2}\left(u_{j, i}^{m}-u_{i, j}^{m}\right) .
\end{array}\right.
$$

The other parameters are

$$
\left\{\begin{array}{l}
g_{1}=\int_{0}^{\infty} \frac{d s}{\left(\alpha_{1}^{2}+s\right) \Delta}, \\
g_{1}^{\prime}=\int_{0}^{\infty} \frac{d s}{\left(\alpha_{2}^{2}+s\right)\left(\alpha_{3}^{2}+s\right) \Delta}, \\
g_{1}^{\prime \prime}=\int_{0}^{\infty} \frac{s d s}{\left(\alpha_{2}^{2}+s\right)\left(\alpha_{3}^{2}+s\right) \Delta}, \\
\Delta^{2}=\left(\alpha_{1}^{2}+s\right)\left(\alpha_{2}^{2}+s\right)\left(\alpha_{3}^{2}+s\right),
\end{array}\right.
$$

where $\alpha_{i}$ denotes the dimensionless axes

$$
\alpha_{i}=a_{i} / a_{0}
$$

and $a_{0}=\left(a_{1} a_{2} a_{3}\right)^{\frac{1}{3}} \cdot \dot{\theta}$ is the angular velocity of the ellipsoid in the space-fixed frame. The other elements of $A_{i j}^{*}$ and the integrals $g_{2}, g_{2}^{\prime} \ldots$ could be obtained by the permutation of the subscripts.

The parameters in (5) and (6) are defined as:

$$
\begin{aligned}
& r_{2}=a_{2} / a_{1}, r_{3}=a_{3} / a_{1}, \\
& z_{1}=\frac{1}{2}\left(r_{2}^{-1}-r_{2}\right), z_{2}=g_{3}^{\prime}\left(\alpha_{1}^{2}+\alpha_{2}^{2}\right), \\
& f_{1}=\left(r_{2}-r_{2}^{-1}\right)^{2}, f_{2}=4 z_{1}^{2}\left(1-2 / z_{2}\right), f_{3}=-4 z_{1} / z_{2},
\end{aligned}
$$

\section{Appendix B. The elastic energy form for capsule}

According to the theoretical model, the element on the membrane is assumed to move along a ellipsoidal shape, then the kinematic equation of membrane can 
be described by [10]

$$
\begin{aligned}
& x_{1}=x_{1}^{0} \cos \phi-\frac{1}{r_{2}} x_{2}^{0} \sin \phi, \\
& x_{2}=x_{2}^{0} \cos \phi+r_{2} x_{1}^{0} \sin \phi, \\
& x_{3}=x_{3}^{0},
\end{aligned}
$$

where $x_{i}^{0}$ defines the initial position of a material point in the membrane at time $t=0$. Hence the deformation gradient tensor is

$$
F_{i j}=\left[\begin{array}{ccc}
\cos \phi & -\frac{\sin \phi}{r_{2}} & 0 \\
r_{2} \sin \phi & \cos \phi & 0 \\
0 & 0 & 1
\end{array}\right]
$$

The left Cauchy-Green tensor is

$$
V^{2}=F \cdot F^{T}=\left[\begin{array}{ccc}
\cos ^{2} \phi+\frac{\sin ^{2} \phi}{r_{2}^{2}} & r_{2} \sin \phi \cos \phi-\frac{1}{r_{2}} \sin \phi \cos \phi & 0 \\
r_{2} \sin \phi \cos \phi-\frac{1}{r_{2}} \sin \phi \cos \phi & r_{2}^{2} \sin ^{2} \phi+\cos ^{2} \phi & 0 \\
0 & 0 & 1
\end{array}\right]
$$

Then we set $\operatorname{det}\left|V^{2}\right|=0$,

$$
\begin{aligned}
\underline{\lambda}^{2}-\left(2 \cos ^{2} \phi+\frac{\sin ^{2} \phi}{r_{2}^{2}}+r_{2}^{2} \sin ^{2} \phi\right) \underline{\lambda} & +\left(\cos ^{2} \phi+\frac{\sin ^{2} \phi}{r_{2}^{2}}\right)\left(r_{2}^{2} \sin ^{2} \phi+\cos ^{2} \phi\right) \\
& -4 z_{1} \sin ^{2} \phi \cos ^{2} \phi=0 .
\end{aligned}
$$

It yields

$$
\begin{aligned}
\lambda_{1}^{2}+\lambda_{2}^{2} & =r_{2}^{2} \sin ^{2} \phi+\frac{\sin ^{2} \phi}{r_{2}^{2}}+2 \cos ^{2} \phi \\
& =\left(r_{2}^{2}+\frac{1}{r_{2}^{2}}-2\right) \sin ^{2} \phi+2 \\
& =4 z_{1}^{2} \sin ^{2} \phi+2,
\end{aligned}
$$




$$
\begin{aligned}
\lambda_{1}^{2} \lambda_{2}^{2} & =\left(\cos ^{2} \phi+\frac{\sin ^{2} \phi}{r_{2}^{2}}\right)\left(r_{2}^{2} \sin ^{2} \phi+\cos ^{2} \phi\right)-4 z_{1} \sin ^{2} \phi \\
& =1 .
\end{aligned}
$$

The first and second strain invariants $I_{1}$ and $I_{2}$ are

$$
\begin{aligned}
& I_{1}=\lambda_{1}^{2}+\lambda_{2}^{2}-2=4 z_{1}^{2} \sin ^{2} \phi, \\
& I_{2}=\lambda^{2} \lambda^{2}-1=0 .
\end{aligned}
$$

For the neo-Hookean Law [17, 33], we have

$$
\begin{aligned}
W^{N H} & =\frac{1}{6} E\left(I_{1}-1+\frac{1}{I_{2}+1}\right) \\
& =\frac{1}{6} E I_{1} \\
& =\frac{2}{3} z_{1}^{2} E \sin ^{2} \phi .
\end{aligned}
$$

\section{References}

[1] M. Abkarian, M. Faivre, A. Viallat, Swinging of red blood cells under shear flow, Physical review letters 98 (18) (2007) 188302.

[2] P. Bagchi, R. M. Kalluri, Dynamic rheology of a dilute suspension of elastic capsules: effect of capsule tank-treading, swinging and tumbling, Journal of Fluid Mechanics 669 (2011) 498-526.

[3] S. Kessler, R. Finken, U. Seifert, Swinging and tumbling of elastic capsules in shear flow, Journal of Fluid Mechanics 605 (2008) 207-226.

[4] D. Cordasco, P. Bagchi, Intermittency and synchronized motion of red blood cell dynamics in shear flow, Journal of Fluid Mechanics 759 (2014) $472-488$.

[5] C. de Loubens, J. Deschamps, G. Boedec, M. Leonetti, Stretching of capsules in an elongation flow, a route to constitutive law, Journal of Fluid Mechanics 767 (2015) R3. 
[6] Y. Sui, X. Chen, Y. Chew, P. Roy, H. Low, Numerical simulation of capsule deformation in simple shear flow, Computers \& Fluids 39 (2) (2010) 242250.

[7] Y. Sui, H. Low, Y. Chew, P. Roy, A front-tracking lattice boltzmann method to study flow-induced deformation of three-dimensional capsules, Computers \& Fluids 39 (3) (2010) 499-511.

[8] H. Ye, H. Huang, X.-Y. Lu, Numerical study on dynamic sorting of a compliant capsule with a thin shell, Computers \& Fluids 114 (2015) 110120.

[9] J. Skotheim, T. Secomb, Red blood cells and other nonspherical capsules in shear flow: oscillatory dynamics and the tank-treading-to-tumbling transition, Physical review letters 98 (7) (2007) 078301.

[10] S. R. Keller, R. Skalak, Motion of a tank-treading ellipsoidal particle in a shear flow, Journal of Fluid Mechanics 120 (1982) 27-47.

[11] D. Barthes-Biesel, Motion of a spherical microcapsule freely suspended in a linear shear flow, Journal of Fluid Mechanics 100 (04) (1980) 831-853.

[12] D. Barthes-Biesel, J. Rallison, The time-dependent deformation of a capsule freely suspended in a linear shear flow, Journal of Fluid Mechanics 113 (1981) 251-267.

[13] K. Chang, W. Olbricht, Experimental studies of the deformation of a synthetic capsule in extensional flow, Journal of Fluid Mechanics 250 (1993) 587-608.

[14] S. Ramanujan, C. Pozrikidis, Deformation of liquid capsules enclosed by elastic membranes in simple shear flow: large deformations and the effect of fluid viscosities, Journal of Fluid Mechanics 361 (1998) 117-143.

[15] Z. Wang, Y. Sui, P. D. Spelt, W. Wang, Three-dimensional dynamics of oblate and prolate capsules in shear flow, Physical Review E 88 (5) (2013) 053021 . 
[16] Y. Sui, Y. Chew, P. Roy, Y. Cheng, H. Low, Dynamic motion of red blood cells in simple shear flow, Physics of Fluids (1994-present) 20 (11) (2008) 112106.

[17] T. Gao, H. H. Hu, P. Ponte Castañeda, Dynamics and rheology of elastic particles in an extensional flow, Journal of Fluid Mechanics 715 (2013) $573-596$.

[18] R. Finken, U. Seifert, Wrinkling of microcapsules in shear flow, arXiv preprint cond-mat/0601589.

[19] A. Walter, H. Rehage, H. Leonhard, Shear induced deformation of microcapsules: shape oscillations and membrane folding, Colloids and Surfaces A: Physicochemical and Engineering Aspects 183 (2001) 123-132.

[20] D. Barthès-Biesel, Capsule motion in flow: Deformation and membrane buckling, Comptes Rendus Physique 10 (8) (2009) 764-774.

[21] T. M. Fischer, Shape memory of human red blood cells, Biophysical journal 86 (5) (2004) 3304-3313.

[22] P. Bagchi, R. M. Kalluri, Dynamics of nonspherical capsules in shear flow, Physical Review E 80 (1) (2009) 016307.

[23] R. Finken, S. Kessler, U. Seifert, Micro-capsules in shear flow, Journal of Physics: Condensed Matter 23 (18) (2011) 184113.

[24] H. Noguchi, Dynamic modes of microcapsules in steady shear flow: Effects of bending and shear elasticities, Physical Review E 81 (5) (2010) 056319.

[25] H. Noguchi, Swinging and synchronized rotations of red blood cells in simple shear flow, Physical Review E 80 (2) (2009) 021902.

[26] P. Vlahovska, Y.-N. Young, G. Danker, C. Misbah, Dynamics of a nonspherical microcapsule with incompressible interface in shear flow, Journal of Fluid Mechanics 678 (2011) 221-247. 
[27] D. Cordasco, A. Yazdani, P. Bagchi, Comparison of erythrocyte dynamics in shear flow under different stress-free configurations, Physics of Fluids (1994-present) 26 (4) (2014) 041902.

[28] Z. Peng, A. Mashayekh, Q. Zhu, Erythrocyte responses in low-shear-rate flows: effects of non-biconcave stress-free state in the cytoskeleton, Journal of Fluid Mechanics 742 (2014) 96-118.

[29] A. Z. Yazdani, P. Bagchi, Phase diagram and breathing dynamics of a single red blood cell and a biconcave capsule in dilute shear flow, Physical Review E 84 (2) (2011) 026314.

[30] J. Deschamps, V. Kantsler, E. Segre, V. Steinberg, Dynamics of a vesicle in general flow, Proceedings of the National Academy of Sciences 106 (28) (2009) 11444-11447.

[31] G. B. Jeffery, The motion of ellipsoidal particles immersed in a viscous fluid, Proceedings of the Royal Society of London. Series A, Containing Papers of a Mathematical and Physical Character (1922) 161-179.

[32] R. Roscoe, On the rheology of a suspension of viscoelastic spheres in a viscous liquid, Journal of Fluid Mechanics 28 (02) (1967) 273-293.

[33] Y. Sui, Y.-T. Chew, P. Roy, H.-T. Low, A hybrid method to study flowinduced deformation of three-dimensional capsules, Journal of Computational Physics 227 (12) (2008) 6351-6371.

[34] T. Gao, H. H. Hu, P. P. Castañeda, Shape dynamics and rheology of soft elastic particles in a shear flow, Physical review letters 108 (5) (2012) 058302 .

[35] Z. Wang, D. Shi, A. Zhang, Three-dimensional lattice boltzmann simulation of bubble behavior in a flap-induced shear flow, Computers \& Fluids 123 (2015) 44-53. 
[36] D. Yu, S. S. Girimaji, Multi-block lattice boltzmann method: extension to $3 \mathrm{~d}$ and validation in turbulence, Physica A: Statistical Mechanics and its Applications 362 (1) (2006) 118-124.

[37] Z. Guo, C. Zheng, B. Shi, Discrete lattice effects on the forcing term in the lattice boltzmann method, Physical Review E 65 (4) (2002) 046308.

[38] S. Kessler, R. Finken, U. Seifert, Elastic capsules in shear flow: Analytical solutions for constant and time-dependent shear rates, The European Physical Journal E: Soft Matter and Biological Physics 29 (4) (2009) 399413.

[39] G. Batchelor, The stress system in a suspension of force-free particles, Journal of fluid mechanics 41 (03) (1970) 545-570.

[40] H. Huang, X. Yang, M. Krafczyk, X.-Y. Lu, Rotation of spheroidal particles in couette flows, Journal of Fluid Mechanics 692 (2012) 369-394. 